\title{
Directional Method of Fundamental Solutions for Three-dimensional Laplace Equation
}

\author{
Chein-Shan Liu ${ }^{1,2}$, Zhuojia $\mathrm{Fu}^{1}$ \& Chung-Lun Kuo ${ }^{1}$ \\ ${ }^{1}$ Center for Numerical Simulation Software in Engineering and Sciences, College of Mechanics and Materials, Hohai \\ University, Nanjing, Jiangsu, China \\ 2 Department of Mechanical and Mechatronic Engineering, National Taiwan Ocean University, Keelung, Taiwan \\ Correspondence: Chung-Lun Kuo, College of Mechanics and Materials, Hohai University, Nanjing, Jiangsu 210098, \\ China. E-mail: eji1215@gmail.com
}

Received: September 13, 2017 Accepted: October 4, 2017 Online Published: November 8, 2017

doi:10.5539/jmr.v9n6p112 URL: https://doi.org/10.5539/jmr.v9n6p112

\begin{abstract}
We propose a simple extension of the two-dimensional method of fundamental solutions (MFS) to a two-dimensional like MFS for the numerical solution of the three-dimensional Laplace equation in an arbitrary interior domain. In the directional MFS (DMFS) the directors are planar orientations, which can take the geometric anisotropy of the problem domain into account, and more importantly the order of the logarithmic singularity with $\ln R$ of the new fundamental solution is reduced than that of the conventional three-dimensional fundamental solution with singularity $1 / r$. Some numerical examples are used to validate the performance of the DMFS.
\end{abstract}

Keywords: three-dimensional Laplace equation, directional method of fundamental solutions, irregular domain

\section{Introduction}

In this paper we extend the method of fundamental solutions (MFS) for the two-dimensional Laplace equation to a MFS for the three-dimensional Laplace equation:

$$
\begin{aligned}
& \Delta u(\mathbf{x})=0, \quad \mathbf{x} \in \Omega \subset \mathbb{R}^{3}, \\
& \left.u\right|_{\mathbf{x} \in \Gamma}=g(\mathbf{x}),
\end{aligned}
$$

where $\Delta$ is the three-dimensional Laplacian operator, and $\Omega$ is an arbitrary three-dimensional bounded domain enclosed by a smooth boundary $\Gamma$.

There are many papers concerned with the numerical solutions of elliptic type boundary value problems by using the meshless and mesh reduction methods, such as Zhu et al., 1998, 1999; Atluri and Zhu, 1998a, 1998b; Atluri et al., 1999; Atluri and Shen, 2002; Li et al., 2007 and Ghimire et al., 2016. The collocation techniques together with the expansion of trial solutions by utilizing different basis-functions were employed to solve the elliptic type boundary value problems; see, for example, Cheng et al., 2003; Hu et al., 2005; Tian et al., 2008, and Hu and Chen, 2008. Li et al. (2008) have given a very detailed description of the collocation Trefftz method.

It is well known that the method of fundamental solutions (MFS) can be used to solve the Laplace equation when a fundamental solution is known (Kupradze and Aleksidze, 1964). Previously, Liu (2008) has proposed a new preconditioner to reduce the ill-condition of the MFS for the Laplace equation. Chen et al. (2015) and Lin et al. (2016) have employed the MFS to solve the three-dimensional modified Helmholtz equation by truncating the off-diagonal terms in the coefficient matrix.

The method of fundamental solutions (MFS) is one of the most powerful meshless techniques that belongs to the category as the boundary-type meshless methods, which has been applied to many engineering problems (Karageorghis and Fairweather, 1998; Tadeu et al., 2010; Lin et al., 2014; Lin et al. 2016). The key idea of the MFS is to represent the solution by a linear combination of fundamental solutions with respect to the source points located outside the domain to avoid the singularity of fundamental solutions. Then the problem is transformed into determining the unknown coefficients by requiring approximations to satisfy the given boundary conditions (Fairweather and Karageorghis, 1998). The details of MFS can be found in the review papers of Fairweather and Karageorghis, 1998; and Golberg and Chen, 1999. A review and a survey on the applications of the MFS to inverse problems are given by Karageorghis et al., 2011.

The singularity happens in the MFS when the source points approach to the physical boundary, which can seriously deteriorate the efficiency and accuracy of the MFS. Therefore, in the MFS a key issue is the reduction of singularity. The 
purpose of this paper is to develop a two-dimensional like MFS in terms of directors for the three-dimensional Laplace equation, which can reduce the singularity. The idea of the introduction of directors in the low-dimensional solutions was first developed by Liu (2016a, 2016b) for heat equation and Helmholtz equation, which can extend the two-dimensional Trefftz method to the higher-dimensional Trefftz method. Then, Liu and Kuo (2016) extended the idea to solve the multi-dimensional wave equation by the directional Trefftz method.

The paper is arranged as follows. In Section 2 we introduce directors into the fundamental solution $\ln r$ for the twodimensional Laplace equation. Then, we prove that the new fundamental solution $\ln R$ is a solution of the three-dimensional Laplace equation. According to the concept of equilibrated matrix we introduce a simple multiple-scale directional method of fundamental solutions (DMFS), which solves several examples in Section 3. Finally, we draw some conclusions in Section 4.

\section{The Directional MFS}

We begin with the following three-dimensional Laplace equation:

$$
\Delta u(x, y, z)=\frac{\partial^{2} u}{\partial x^{2}}+\frac{\partial^{2} u}{\partial y^{2}}+\frac{\partial^{2} u}{\partial z^{2}}=0, \quad(x, y, z) \in \Omega,
$$

where $\Delta$ is the three-dimensional Laplacian operator.

In the potential theory, the method of fundamental solutions (MFS) can be used to solve the Laplacian problems when a fundamental solution is known (Kupradze and Aleksidze, 1964). In the MFS the trial solution of $u$ at the field point $\mathbf{p}=(x, y, z)$ can be expressed as a linear combination of the fundamental solutions $U\left(\mathbf{p}, \mathbf{s}_{j}\right)$ :

$$
u(\mathbf{p})=\sum_{j=1}^{n} c_{j} U\left(\mathbf{p}, \mathbf{s}_{j}\right), \quad \mathbf{s}_{j} \in \Omega^{c},
$$

where $n$ is the number of source points, $c_{j}$ are unknown coefficients, $\mathbf{s}_{j}$ are source points, and $\Omega^{c}$ is the complementary set of $\Omega$. For the Laplace equation (3) we have the fundamental solutions:

$$
U\left(\mathbf{p}, \mathbf{s}_{j}\right)=\frac{1}{r_{j}}, \quad r_{j}=\left\|\mathbf{p}-\mathbf{s}_{j}\right\| .
$$

Now we propose a two-dimensional like method of fundamental solutions (MFS) for the Laplace equation (3). Let $\mathbf{s}=\left(x^{s}, y^{s}, z^{s}\right)$ be a generic source point and

$$
\eta=d_{2}\left(y-y^{s}\right)+d_{3}\left(z-z^{s}\right)
$$

where $\left(d_{2}, d_{3}\right)$ is a director satisfying

$$
d_{2}^{2}+d_{3}^{2}=1
$$

Theorem 1 A directional fundamental solution of the three-dimensional Laplace equation (3) is given by

$$
U(\mathbf{p}, \mathbf{s})=\ln R:=\ln \sqrt{\left(x-x^{s}\right)^{2}+\eta^{2}}=\ln \sqrt{\left(x-x^{s}\right)^{2}+\left[d_{2}\left(y-y^{s}\right)+d_{3}\left(z-z^{s}\right)\right]^{2}},
$$

which means that $U$ is a solution of Eq. (3).

Proof. First we have

$$
\begin{aligned}
& \frac{\partial U}{\partial x}=\frac{x-x^{s}}{\left(x-x^{s}\right)^{2}+\eta^{2}}=\frac{x-x^{s}}{\left(x-x^{s}\right)^{2}+\left[d_{2}\left(y-y^{s}\right)+d_{3}\left(z-z^{s}\right)\right]^{2}}, \\
& \frac{\partial U}{\partial y}=\frac{d_{2} \eta}{\left(x-x^{s}\right)^{2}+\eta^{2}}=\frac{d_{2}\left[d_{2}\left(y-y^{s}\right)+d_{3}\left(z-z^{s}\right)\right]}{\left(x-x^{s}\right)^{2}+\left[d_{2}\left(y-y^{s}\right)+d_{3}\left(z-z^{s}\right)\right]^{2}}, \\
& \frac{\partial U}{\partial z}=\frac{d_{3} \eta}{\left(x-x^{s}\right)^{2}+\eta^{2}}=\frac{d_{3}\left[d_{2}\left(y-y^{s}\right)+d_{3}\left(z-z^{s}\right)\right]}{\left(x-x^{s}\right)^{2}+\left[d_{2}\left(y-y^{s}\right)+d_{3}\left(z-z^{s}\right)\right]^{2}} .
\end{aligned}
$$

Taking the derivative of each term with respect to $x, y$ and $z$, respectively, we have

$$
\begin{aligned}
& \frac{\partial^{2} U}{\partial x^{2}}=\frac{\left(x-x^{s}\right)^{2}+\eta^{2}-2\left(x-x^{s}\right)^{2}}{\left[\left(x-x^{s}\right)^{2}+\eta^{2}\right]^{2}}, \\
& \frac{\partial^{2} U}{\partial y^{2}}=\frac{d_{2}^{2}\left[\left(x-x^{s}\right)^{2}+\eta^{2}\right]-2 d_{2}^{2} \eta^{2}}{\left[\left(x-x^{s}\right)^{2}+\eta^{2}\right]^{2}}, \\
& \frac{\partial^{2} U}{\partial z^{2}}=\frac{d_{3}^{2}\left[\left(x-x^{s}\right)^{2}+\eta^{2}\right]-2 d_{3}^{2} \eta^{2}}{\left[\left(x-x^{s}\right)^{2}+\eta^{2}\right]^{2}} .
\end{aligned}
$$


Summing the above three terms leads to

$$
\begin{aligned}
& \frac{\partial^{2} U}{\partial x^{2}}+\frac{\partial^{2} U}{\partial y^{2}}+\frac{\partial^{2} U}{\partial z^{2}}=\frac{1}{\left[\left(x-x^{s}\right)^{2}+\eta^{2}\right]^{2}} \\
& \left\{\left(x-x^{s}\right)^{2}+\eta^{2}-2\left(x-x^{s}\right)^{2}+d_{2}^{2}\left[\left(x-x^{s}\right)^{2}+\eta^{2}\right]-2 d_{2}^{2} \eta^{2}+d_{3}^{2}\left[\left(x-x^{s}\right)^{2}+\eta^{2}\right]-2 d_{3}^{2} \eta^{2}\right\} \\
& =\frac{1}{\left[\left(x-x^{s}\right)^{2}+\eta^{2}\right]^{2}}\left\{\eta^{2}-\left(x-x^{s}\right)^{2}+\left(d_{2}^{2}+d_{3}^{2}\right)\left[\left(x-x^{s}\right)^{2}+\eta^{2}\right]-2\left(d_{2}^{2}+d_{3}^{2}\right) \eta^{2}\right\}=0,
\end{aligned}
$$

where we have used $d_{2}^{2}+d_{3}^{2}=1$ specified in Eq. (7). This ends the proof.

Correspondingly, we can expand the trial solution of $u(x, y, z)$ for the three-dimensional Laplace equation (3) by

$$
u(x, y, z)=\sum_{i=1}^{m_{1}} \sum_{j=1}^{m} a_{i j} s_{i j} \ln \sqrt{\left(x-x_{j}^{s}\right)^{2}+\left[d_{2}^{i}\left(y-y_{j}^{s}\right)+d_{3}^{i}\left(z-z_{j}^{s}\right)\right]^{2}},
$$

where $n=m m_{1}$ is the number of unknown coefficients $\left\{a_{i j}\right\}$, and each $\left(d_{2}^{i}, d_{3}^{i}\right)$ is a direction in the plane $(y, z)$, satisfying Eq. (7), which is given by

$$
d_{2}^{i}=\cos \left(2 i \pi / m_{1}\right), \quad d_{3}^{i}=\sin \left(2 i \pi / m_{1}\right) .
$$

In Theorem 1 we can change the bases to other bases by cyclically changing the coordinates $(x, y, z)$. Instead of Eq. (6), we can also use

$$
\begin{array}{ll}
\eta=d_{1}^{j}\left(x-x^{s}\right)+d_{3}^{j}\left(z-z^{s}\right), & R=\left(y-y^{s}\right)^{2}+\eta^{2}, \\
\eta=d_{1}^{j}\left(x-x^{s}\right)+d_{2}^{j}\left(y-y^{s}\right), & R=\left(z-z^{s}\right)^{2}+\eta^{2} .
\end{array}
$$

Inserting the above into Eq. (8) we can derive other two different bases.

\section{Numerical Tests}

Now, we are ready to apply the directional method of fundamental solutions (DMFS) to find the numerical solutions of three-dimensional Laplace equation.

\subsection{Example 1}

In order to demonstrate the performance of DMFS we give a solution of the 3D Laplace equation:

$$
u(x, y, z)=x y z+x^{2}-\frac{y^{2}+z^{2}}{2}
$$

defined in a domain $\Omega$ with boundary given by the following parametric equation:

$$
\Gamma=\{(x, y, z) \mid x=\rho \cos \theta, y=\rho \sin \theta \sin \phi, z=\rho \sin \theta \cos \phi, 0 \leq \theta \leq 2 \pi, 0 \leq \phi \leq \pi\},
$$

where

$$
\rho(\theta)=\left[\cos (3 \theta)+\sqrt{8-\sin ^{2}(3 \theta)}\right]^{\frac{1}{3}}
$$

and the problem domain is shown in Fig. 1(a).

In the DMFS we collocate $n_{q}$ uniformly distributed points on the surface $\Gamma$ to satisfy the specified boundary conditions, while $m$ source points are uniformly distributed on a surface with a distance $D_{0}$ from $\Gamma$. The resulting linear system is scaled by $s_{i j}$, such that each column norm is equal to $S_{0}$, which is known as the equilibrated matrix method (Liu and Atluri, 2013), and then we solve the scaled linear system by using the conjugate gradient method (CGM) under a given convergence criterion $\varepsilon$. The dimension of the normal system is $n$.

Under $m=25, m_{1}=5$ (hence, $n=125$ ), $n_{q}=900, S_{0}=0.001$ and $D_{0}=9$, the DMFS is convergent with 3107 steps under $\varepsilon=10^{-10}$. When we compare the numerical and exact solutions of $u$ on a surface $S:=\{(x, y, z) \mid x=\rho / 2 \cos \theta, y=$ $\rho / 2 \sin \theta \sin \phi, z=\rho / 2 \sin \theta \cos \phi, 0 \leq \theta \leq 2 \pi, 0 \leq \phi \leq \pi\}$ inside $\Omega$, the error is plotted in Fig. 2(a), whose maximum error is $2.01 \times 10^{-6}$. The numerical solution obtained by the DMFS is very accurate even in an irregular domain.

The presented DMFS is not sensitive to the distribution of source points. For example, when we increase $D_{0}=9$ to $D_{0}=20$ and under other same parameters values, the accuracy is slightly reduced to $8.3 \times 10^{-6}$, but the convergence speed is slightly faster with 2649 steps. 
When we apply the conventional MFS to solve this problem, under $D_{0}=10$ and other parameters the same, it does not converge within 30000 steps, and the maximum error on $S$ raises to $7.09 \times 10^{-6}$ as shown in Fig. 2(b). It can be seen that for this problem the DMFS is better than the MFS, no matter the efficiency or the accuracy.

\subsection{Example 2}

We consider the same exact solution as that in Example 1; however, the domain as shown in Fig. 1(b) is bounded by a torus, given by

$$
(x, y, z)=[(a+b \cos \phi) \cos \theta,(a+b \cos \phi) \sin \theta, b \sin \phi], \quad 0 \leq \theta, \phi \leq 2 \pi,
$$

where we fix $a=5$ and $b=1$.

To take the geometric anisotropy of the problem domain into account, we employ Eq. (19) as the bases of the DMFS. Under $m=64, m_{1}=10(n=640), n_{q}=900, S_{0}=1$ and $D_{0}=10$, where the source points are uniformly distributed on a sphere with a radius $D_{0}$, the DMFS does not converge within 30000 steps under $\varepsilon=10^{-10}$. In Figs. 3(a) and 3(b) we compare the numerical and exact solutions of $u$ on a surface $S:=\{(x, y, z)=[(a+b / 2 \cos \phi) \cos \theta,(a+b / 2 \cos \phi) \sin \theta, b / 2 \sin \phi]\}$ inside $\Omega$, of which the maximum error is $6.57 \times 10^{-2}$. Upon knowing the maximum value of $|u|$ is about 30 , the accuracy is acceptable.

When we apply the conventional MFS to solve this problem, under $D_{0}=10, \varepsilon=10^{-10}$, and $m=25(n=625)$, it does not converge within 30000 steps, and the maximum error on $S$ raises to 0.64 . It can be seen that for this problem the DMFS is accurate than the MFS. From Figs. 3(b) and 3(c) we can observe that the numerical solution obtained by the MFS is severely distorted from the one of the exact solution.

\subsection{Example 3}

We give a complex 3D Laplacian problem with:

$$
\rho(\theta, \phi)=(2+\cos \theta)\left[\cos (3 \phi)+\sqrt{8-\sin ^{2}(3 \phi)}\right]^{\frac{1}{3}},
$$

where $\rho$ is the radius function of the boundary contour of $\Omega$ in the spherical coordinates $(r \leq \rho, 0 \leq \theta \leq 2 \pi, 0 \leq \phi \leq \pi)$. The problem domain is shown in Fig. 1(c). We consider the same exact solution as that in Example 1.

Under $m=25, m_{1}=8(n=200), n_{q}=900, S_{0}=0.001$ and $D_{0}=25$, the DMFS converges after 12539 steps under $\varepsilon=$ $10^{-9}$. When we compare the numerical and exact solutions of $u$ on a surface $S:=\{(x, y, z)=(\rho / 2 \sin \phi \cos \theta, \rho / 2 \sin \phi \sin \theta, \rho / 2 \cos \phi)\}$ inside $\Omega$, the error is plotted in Fig. 4, of which the maximum error is $1.83 \times 10^{-4}$.

\subsection{Example 4}

Then, we give a solution of the 3D Laplace equation:

$$
u(x, y, z)=e^{y} \cos x+e^{x} \sin z
$$

defined in a bumpy sphere as shown in Fig. 1(d), with its boundary given by the following parametric equation:

$$
\Gamma=\{(x, y, z) \mid x=\rho \cos \theta \sin \phi, y=\rho \sin \theta \sin \phi, z=\rho \cos \phi, 0 \leq \theta \leq 2 \pi, 0 \leq \phi \leq \pi\},
$$

where

$$
\rho(\theta, \phi)=1+\frac{1}{6} \sin 7 \theta \sin 6 \phi .
$$

Under $m=36, m_{1}=15(n=540), n_{q}=900, S_{0}=1$ and $D_{0}=10$, the DMFS does not converge within 30000 steps under $\varepsilon=10^{-10}$. In Fig. 5(a) we plot the numerical solution of $u$ on a surface $S:=\{(x, y, z) \mid x=\rho / 2 \cos \theta \sin \phi, y=$ $\rho / 2 \sin \theta \sin \phi, z=\rho / 2 \cos \phi, 0 \leq \theta \leq 2 \pi, 0 \leq \phi \leq \pi\}$, of which the maximum error is $4.58 \times 10^{-5}$. It can be seen that the solution on the surface $S$ is very complex. The accuracy is good.

\subsection{Example 5}

In order to further demonstrate the performance of DMFS we give a complex solution:

$$
u(x, y, z)=\cosh z \cos (\sqrt{2} x) \sinh y+\sinh z \sin (\sqrt{2} x) \cosh y
$$

defined in the bumpy sphere as that in Example 4.

Under $m=36, m_{1}=15(n=540), n_{q}=900, S_{0}=1$ and $D_{0}=10$, the DMFS does not converge within 30000 steps under $\varepsilon=10^{-10}$. In Fig. 6 we compare the numerical solution and exact solution of $u$ on the surface $S:=\{(x, y, z) \mid x=$ $\rho / 2 \cos \theta \sin \phi, y=\rho / 2 \sin \theta \sin \phi, z=\rho / 2 \cos \phi, 0 \leq \theta \leq 2 \pi, 0 \leq \phi \leq \pi\}$, of which the maximum error is $8.54 \times 10^{-5}$. It can be seen that the numerical solution on the surface $S$ is accurate. 


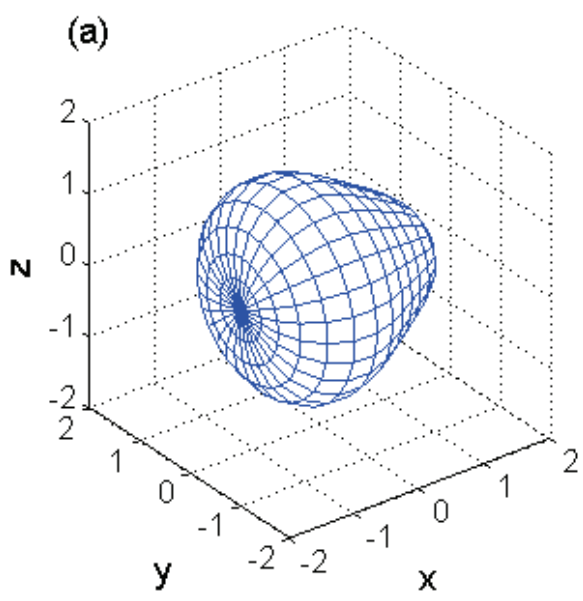

(b)
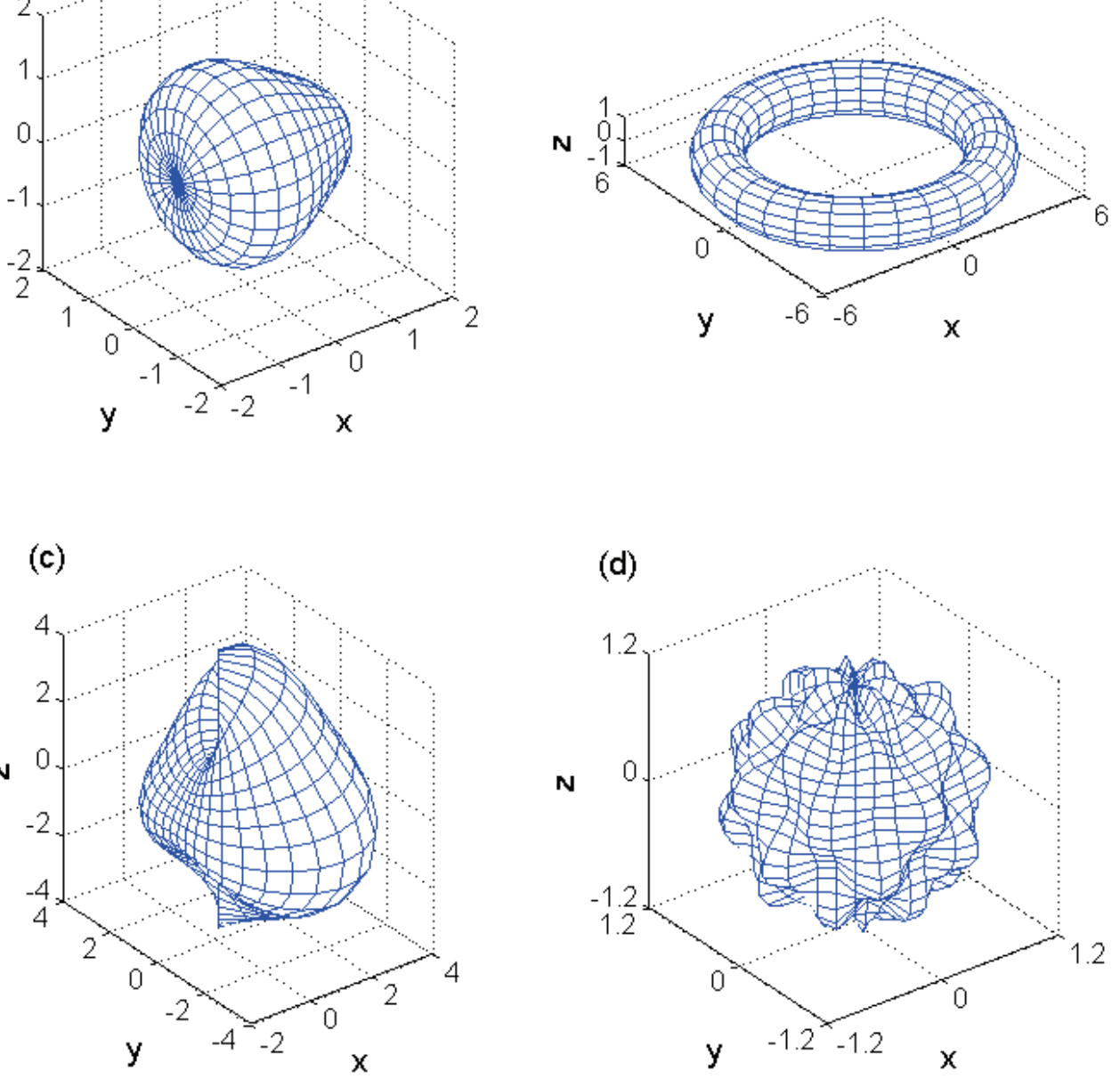

Figure 1. The problem domains for Examples 1, 2, 3 and 4 

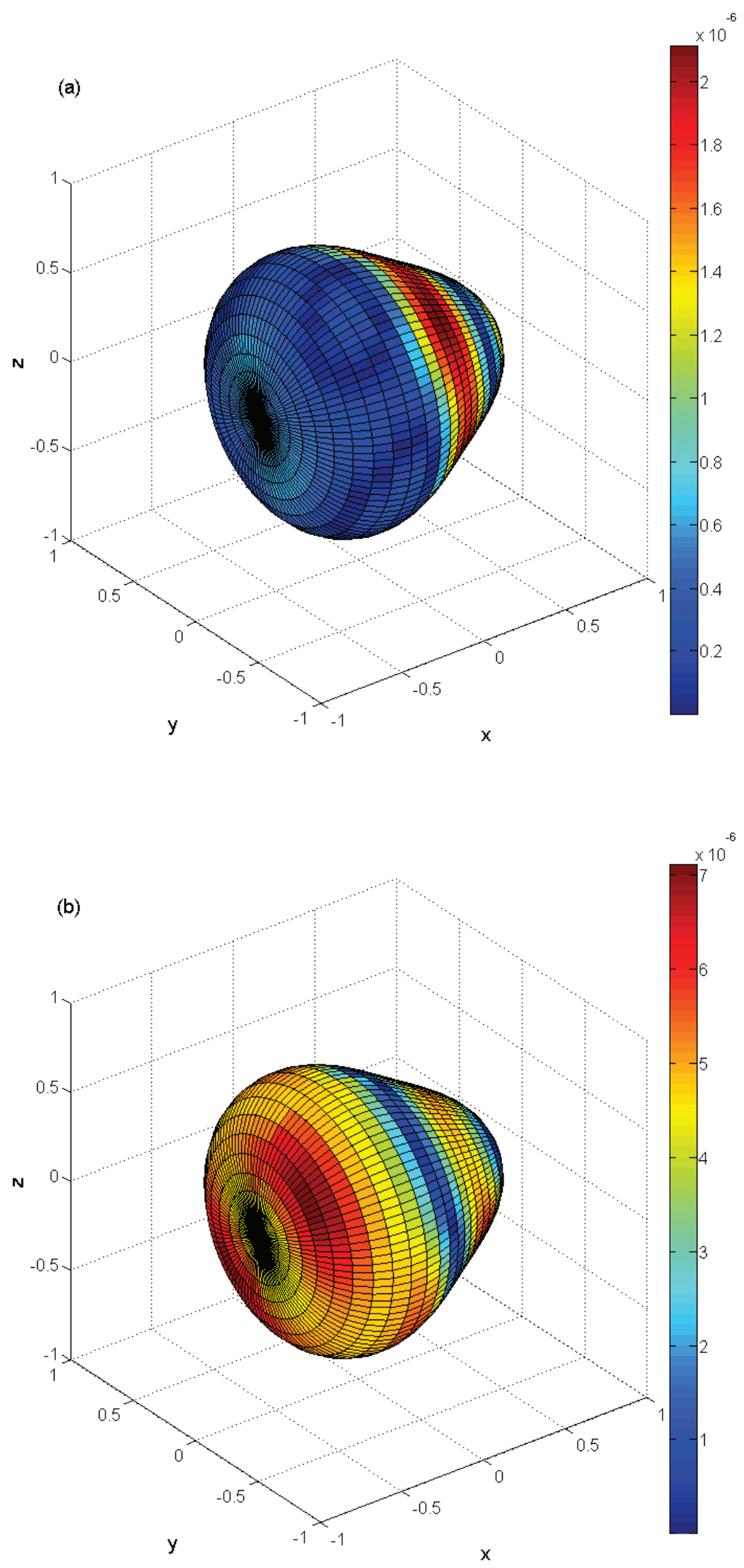

Figure 2. The errors of Example 1 over the parametric plane, (a) DMFS, and (b) MFS 


$$
\begin{aligned}
& 0 \\
& 0
\end{aligned}
$$




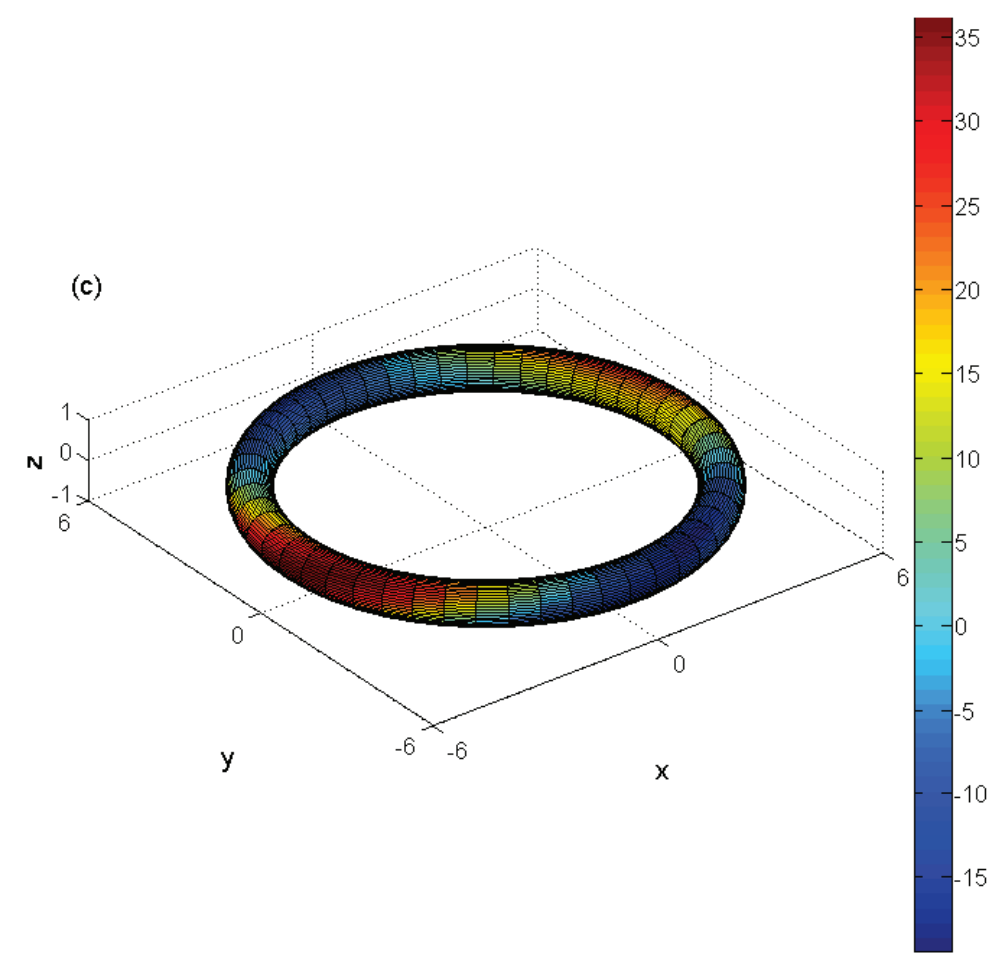

Figure 3. For Example 2 over the parametric plane, comparing (a) numerical solution of the DMFS, (b) exact solution, and (c) numerical solution of the MFS

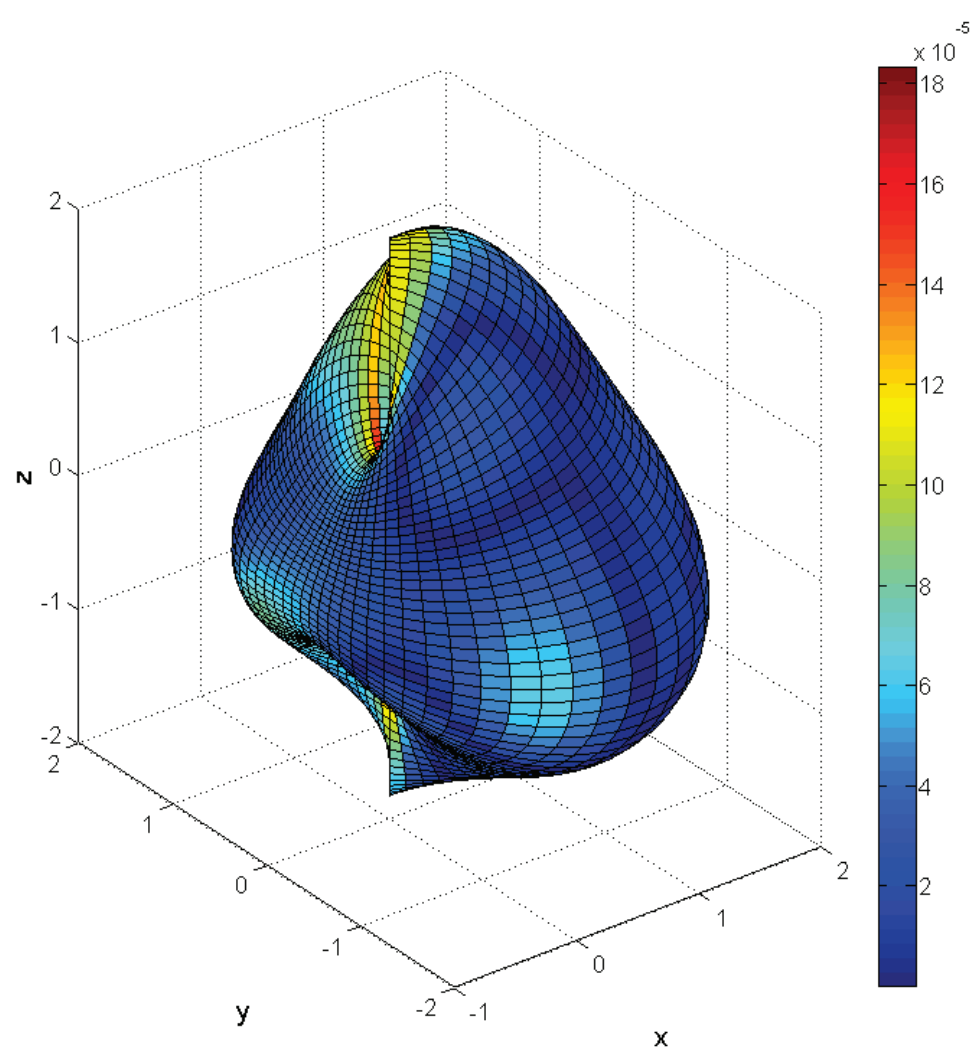

Figure 4. For Example 3 showing numerical errors of the DMFS over the parametric plane 

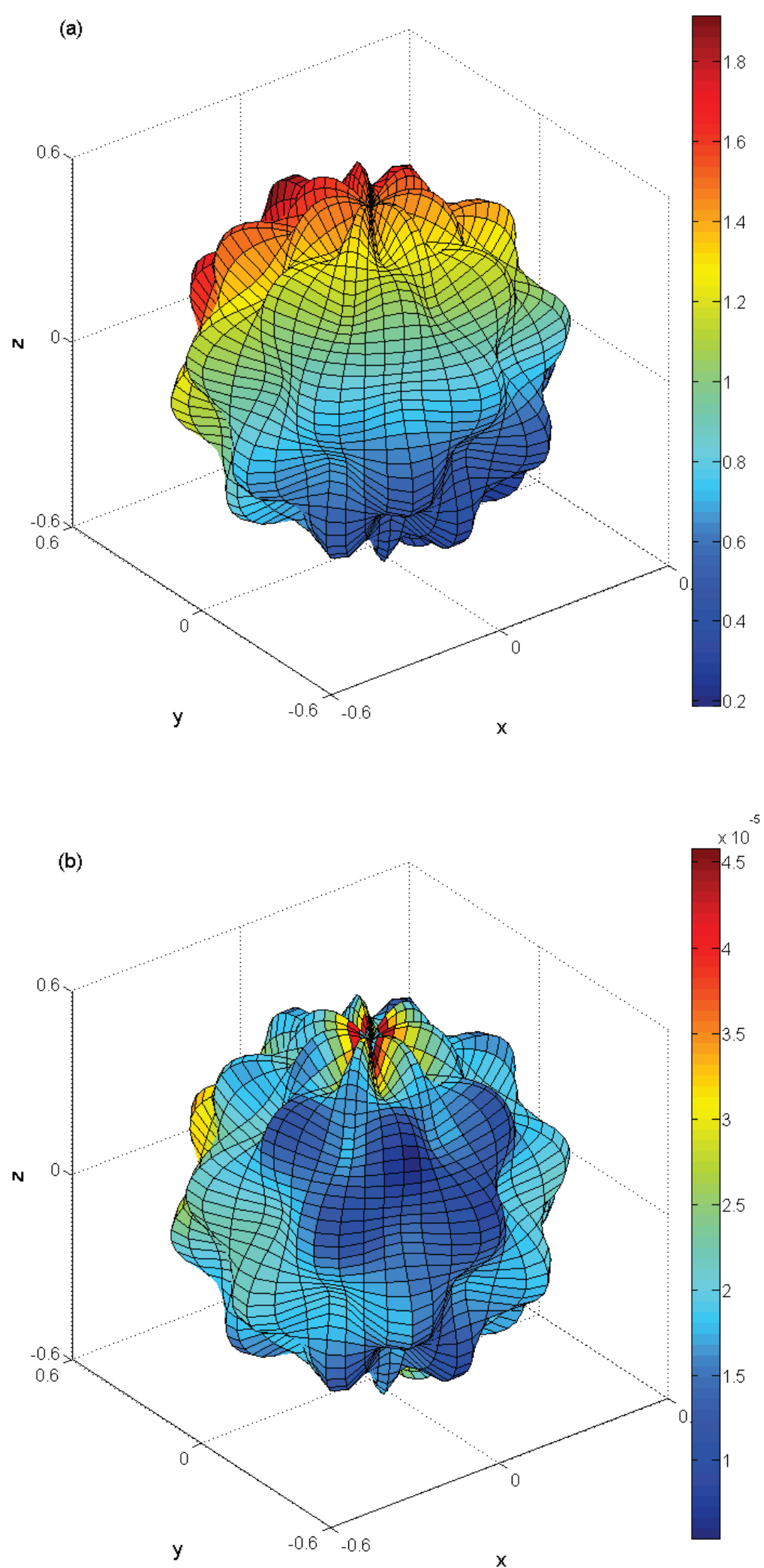

Figure 5. For Example 4, (a) plotting numerical solution, and (b) showing numerical errors of the DMFS over the parametric plane 

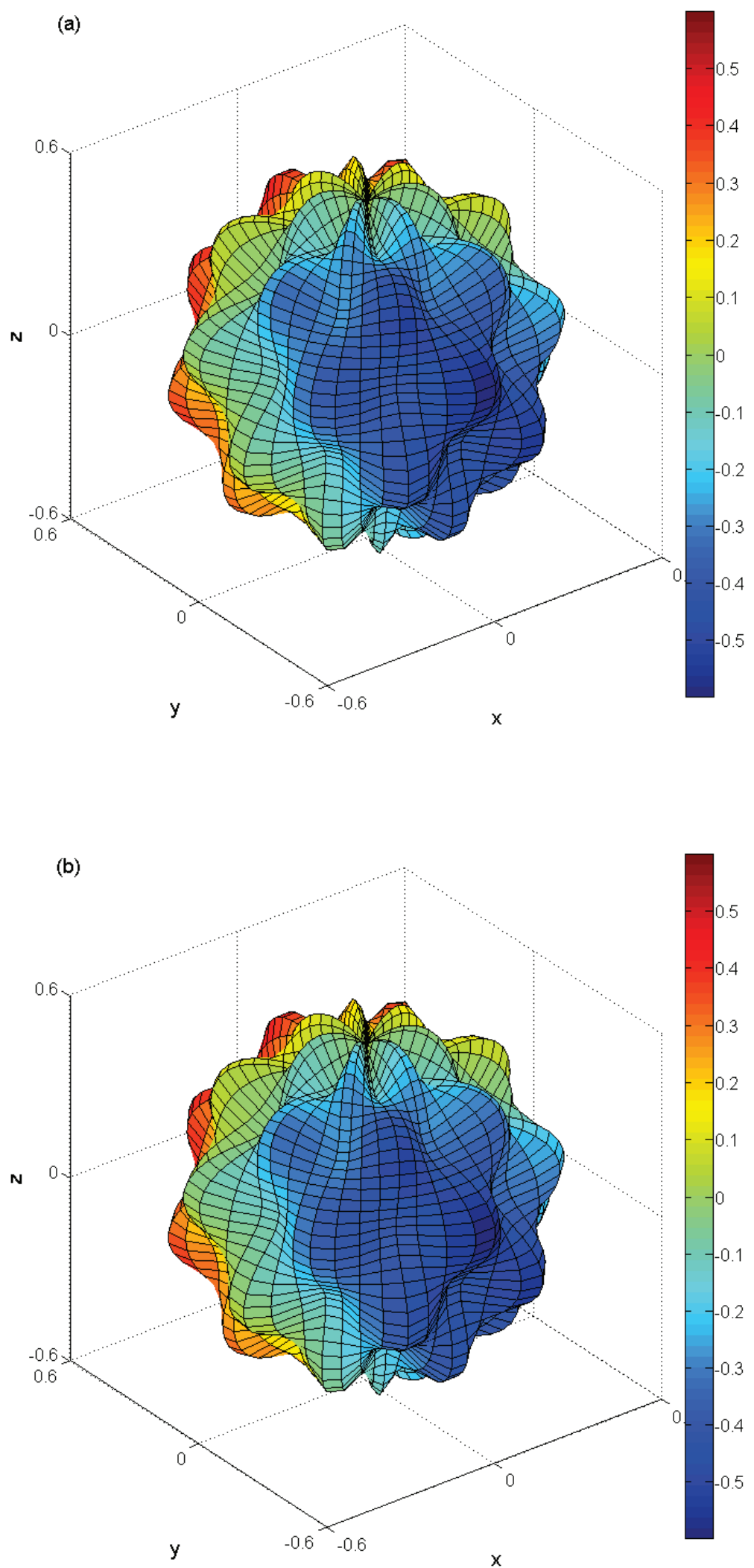

Figure 6. For Example 5, comparing (a) numerical solution of the DMFS, and (b) exact solution 


\section{Conclusions}

We have proposed by using a two-dimensional like MFS to treat the Dirichlet problems of the three-dimensional Laplace equation in arbitrary domain. The directors used in the two-dimensional MFS resulted to a novel method, namely the directional method of fundamental solutions (DMFS) for the three-dimensional Laplace equation, of which the singularity is reduced from $1 / r$ of the conventional MFS to a logarithmic singularity $\ln R$. We found that the new DMFS gains three advantages than the original MFS due to the reduction of singularity: raising the accuracy, increasing the efficiency, and also being not sensitive to the distribution of source points. We have performed five examples to assess the performance of the DMFS.

\section{Acknowledgements}

The Fundamental Research Funds for the Central Universities under the Grant Number 2017B05714 for the financial support to the first and third authors is highly appreciated.

\section{References}

Atluri, S. N., \& Shen, S. (2002). The meshless local Petrov-Galerkin (MLPG) method: a simple \& less-costly alternative to the finite element and boundary element methods. Comput. Model. Eng. Sci., 3, 11-51.

Atluri, S. N., \& Zhu, T. L. (1998a). A new meshless local Petrov-Galerkin (MLPG) approach in computational mechanics. Comput. Mech., 22, 117-127. https://doi.org/10.1007/s004660050346

Atluri, S. N., \& Zhu, T. L. (1998b). A new meshless local Petrov-Galerkin (MLPG) approach to nonlinear problems in computer modeling and simulation. Comput. Model. Simul. Eng., 3, 187-196.

Atluri, S. N., Kim, H. G., \& Cho, J. Y. (1999). A critical assessment of the truly meshless local Petrov-Galerkin (MLPG), and local boundary integral equation (LBIE) methods. Comput. Mech., 24, 348-372. https://doi.org/10.1007/s004660050457

Chen, C. S., Jiang, X., Chen, W., \& Yao, G. (2015). Fast solution for solving the modified Helmholtz equation with the method of fundamental solutions. Commun. Comput. Phys., 4, 867-886.

Cheng, A. H. D., Golberg, M. A., Kansa, E.J., \& Zammito G. (2003). Exponential convergence and H-c multiquadric collocation method for partial differential equations. Num. Meth. Part. Diff. Eqs., 19, 571-594. https://doi.org/10.1002/num.10062

Fairweather, G., \& Karageorghis, A. (1998). The method of fundamental solutions for elliptic boundary value problems. Adv. Comput. Math., 9, 69-95.

Ghimire, B. K., Tian, H. Y., \& Lamichhane, A. R. (2016). Numerical solutions of elliptic partial differential equations using Chebyshev polynomials. Comput. Math. Appl., 72, 1042-1054. https://doi.org/10.1016/j.camwa.2016.06.012

Golberg, M. A., \& Chen, C. S. (1999). The method of fundamental solutions for potential, Helmholtz and diffusion problems. In: Boundary integral methods: numerical and mathematical aspects. Computational Engineering,vol.1, WIT Press/Comput. Mech. Publ., Boston, MA, 103-176.

Hu, H. Y., \& Chen, J. S. (2008). Radial basis collocation method and quasi-Newton iteration for nonlinear elliptic problems. Num. Meth. Part. Diff. Eqs., 24, 991-1017. https://doi.org/10.1002/num.20303

Hu, H. Y., Li, Z. C., \& Cheng, A. H. D. (2005). Radial basis collocation methods for elliptic boundary value problems. Comput. Math. Appl., 50, 289-320. https://doi.org/10.1016/j.camwa.2004.02.014

Karageorghis, A., \& Fairweather, G. (1998). The method of fundamental solutions for axisymmetric acoustic scattering and radiation problems. J. Acoust. Soc. Am., 104, 3212-3218. http://dx.doi.org/10.1121/1.423961

Karageorghis, A., Lesnic, D., \& Marin, L. (2011). A survey of applications of the MFS to inverse problems. Inv. Prob. Sci. Eng., 19, 309-336. http://dx.doi.org/10.1080/17415977.2011.551830

Kupradze, V. D., \& Aleksidze, M. A. (1964). The method of functional equations for the approximate solution of certain boundary value problems. USSR Comput. Math. Math. Phy., 4, 82-126. https://doi.org/10.1016/00415553(64)90006-0

Li, Z. C., Lu, T. T., Huang, H. T., \& Cheng, A. H. D. (2007). Trefftz, collocation, and other boundary methods-A comparison. Num. Meth. Part. Diff. Eqs., 23, 93-144. https://doi.org/10.1002/num.20159

Li, Z. C., Lu, T. T., Huang, H. T., \& Cheng, A. H. D. (2008). Trefftz and Collocation Methods. Southampton, WIT Press.

Lin, J., Chen, C. S., \& Liu, C.-S. (2016). Fast solution of three-dimensional modified Helmholtz equations by the method 
of fundamental solutions. Commun. Comput. Phys., 20, 512-533. https://doi.org/10.4208/cicp.060915.301215a

Lin, J., Chen, W., \& Chen, C. S. (2014). A new scheme for the solution of reaction diffusion and wave propagation problems. Appl. Math. Model., 38, 5651-5664. https://doi.org/10.1016/j.apm.2014.04.060

Lin, J., Chen, C. S., Liu, C.-S., \& Lu, J. (2016). Fast simulation of multi-dimensional wave problems by the sparse scheme of the method of fundamental solutions. Comput. Math. Appl., 72, 555-567. https://doi.org/10.1016/j.camwa.2016.05.016

Liu, C.-S. (2008). Improving the ill-conditioning of the method of fundamental solutions for 2D Laplace equation. Comput. Model. Eng. Sci., 28, 77-93.

Liu, C.-S. (2016a). A multiple/scale/direction polynomial Trefftz method for solving the BHCP in high-dimensional arbitrary simply-connected domains. Int. J. Heat Mass Transfer., 92, 970-978. https://doi.org/10.1016/j.ijheatmasstransfer.2015.09.057

Liu, C.-S. (2016b). A simple Trefftz method for solving the Cauchy problems of three-dimensional Helmholtz equation. Eng. Anal. Bound. Elem., 63, 105-113. https://doi.org/10.1016/j.enganabound.2015.11.009

Liu, C.-S., \& Atluri, S. N. (2013). Numerical solution of the Laplacian Cauchy problem by using a better postconditioning collocation Trefftz method. Eng. Anal. Bound. Elem., 37, 74-83. https://doi.org/10.1016/j.enganabound.2012.08.008

Liu, C.-S., \& Kuo, C. L. (2016). A multiple-direction Trefftz method for solving the multi-dimensional wave equation in an arbitrary spatial domain. J. Comput. Phys., 321, 39-54. https://doi.org/10.1016/j.jcp.2016.05.030

Tadeu, A., Simoes, N., \& Simoes, I. (2010). Coupling BEM/TBEM and MFS for the simulation of transient conduction heat transfer. Int. J. Numer. Meth. Eng., 84, 179-213. https://doi.org/10.1002/nme.2891

Tian, H. Y., Reutskiy, S., \& Chen, C. S. (2008). A basis function for approximation and the solutions of partial differential equations. Num. Meth. Part. Diff. Eqs., 24, 1018-1036. https://doi.org/10.1002/num.20304

Zhu, T., Zhang, J., \& Atluri, S. N. (1998). A meshless local boundary integral equation (LBIE) method for solving nonlinear problems. Comput. Mech., 22, 174-186. https://doi.org/10.1007/s004660050351

Zhu, T., Zhang, J., \& Atluri, S. N. (1999). A meshless numerical method based on the local boundary integral equation (LBIE) to solve linear and non-linear boundary value problems. Eng. Anal. Bound. Elem., 23, 375-389. https://doi.org/10.1016/S0955-7997(98)00096-4

\section{Copyrights}

Copyright for this article is retained by the author(s), with first publication rights granted to the journal.

This is an open-access article distributed under the terms and conditions of the Creative Commons Attribution license (http://creativecommons.org/licenses/by/4.0/). 